\title{
Seguimiento de las recomendaciones sobre psicofarmacología y su repercusión conductual en la discapacidad intelectual.
}

\author{
Expert psychopharmacologic recommendations follow-up and its behavioral impact on \\ intellectual disability patients.
}

\begin{abstract}
M. Jiménez Cubero a , V. Aguilar Noguera ${ }^{\text {b }}$, V. Pol Viedma ${ }^{c}$, G. Mata García $^{\mathrm{d}}$, S. Crespo Valera ${ }^{\mathrm{e}}$, F. Martínez Granados f , A. Carratalá Marco ${ }^{\text {, }}$, E. Pérez Martínez ${ }^{\text {h }}$, Emilio Pol Yanguas i

${ }^{a}$ Enfermero/a, Centro San Rafael para discapacitados intelectuales. Alicante, España. ${ }^{b}$ Enfermero/a, Centro San Rafael para discapacitados intelectuales. Alicante, España. ${ }^{c}$ Enfermera, Hospital General de Alicante. Alicante, España. ${ }^{d}$ Psicóloga, Centro San Rafael para discapacitados intelectuales. Alicante, España. ${ }^{e}$ Psi-

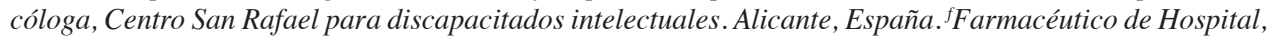
Centro Dr. Esquerdo para enfermos mentales. Alicante, España. ${ }^{g}$ Médico, Directora del Centro San Rafael para discapacitados intelectuales. Alicante, España. ${ }^{h} P$ siquiatra, Hospital General de Alicante. Alicante, España. ${ }^{i}$ Farmacéutico de Hospital, Centro Dr. Esquerdo para enfermos mentales. Alicante, España.
\end{abstract}

Correspondencia: Emilio Pol Yanguas (epol@dip-alicante.es)

Recibido: 27/07/2012; aceptado con modificaciones: 25/01/2013

RESUMEN: En la población con discapacidad intelectual (DI) hay una elevada morbilidad psiquiátricaconductual. Se estima que por este motivo entre $1 / 3$ y 3/4 de estas personas reciben antipsicóticos. Existe un consenso de expertos para guiar la toma de decisiones farmacoterapéuticas en estos casos. Su aplicación conseguiría una mayor eficacia del tratamiento, reduciendo los problemas conductuales, mejorando las habilidades adaptativas. El "Inventory for client and agency planning" (ICAP) es un instrumento para valoración y evaluación de servicios para personas con DI, que incluye escalas para puntuación de problemas conductuales y de conductas adaptativas. Para determinar la asociación entre el seguimiento de las recomendaciones farmacoterapeuticas de los expertos y las puntuaciones de los problemas conductuales y las habilidades adaptativas en un grupo de sujetos con DI, se realizó un estudio observación transversal. El tratamiento farmacológico recibido por cada sujeto de un colectivo de sujetos diagnosticados de DI (CIE-10) se clasificó como conforme o no con las recomendaciones de la guía en lo referente a los criterios de indicación, dosis, duración y polifarmacia. Se compararon las puntuaciones de conducta adaptativa y de problemas de conducta del ICAP en función de la conformidad del tratamiento con los criterios.

El cumplimiento del criterio de dosis se asoció con mejor conducta adaptativa $(\mathrm{p}<0,05)$, el cumplimiento de los criterios de duración y polifarmacia se asocia-
ABSTRACT: Subjects with Intellectual Disability (ID) are frequently affected by behavioral and psychiatric co-mobility. As a consequence between $1 / 3$ and $3 / 4$ are under antipsychotic treatment. It is available an expert consensus guideline with the purpose of guiding pharmacological treatment decisions. The achievement of expert recommendations may optimize drug therapy efficacy and reduce behavioral problems as well as enhance adaptive abilities. The "Inventory for client and agency planning" (ICAP) is a psychometric tool designed to assess health care to ID patients, and includes items that grade behavioral and adaptive problems.

We designed a transversal study in order to determine the association between the follow-up of expert recommendations and the scores achieved on behavioral and adaptive problems items. Drug therapy been prescribed to patients diagnosed of ID (CIE-10) was analyzed and classified into a dichotomous "Do" or "Do not" achieve guideline recommendations in regard of several pharmaceutical aspects such as drug therapy indication, dosage, treatment duration and poly-pharmacy. Scores from ICAP tool as compared to guideline compliance. Follow-up of dose criteria was associated to a better adaptive behavior $(\mathrm{p}<0,05)$ and follow-up of poly-pharmacy criteria and duration treatment criteria was associated better behavioral out- 
ron con menores problemas de conducta $(\mathrm{p}<0,05)$. No hubo asociación entre cumplimiento del criterio de indicación con la puntuación de problemas de conducta, ni de las habilidades adaptativas. PALABRAS CLAVE: Discapacidad intelectual, Trastorno de conducta, Adaptación psicológica, Farmacoterapia, Guía de práctica. comes $(\mathrm{p}<0,05)$. We found no association between follow-up of indication criteria and behavioral or adaptive problems.

KEY WORDS: Intellectual Disability; Behavioral symptoms; Adaptation, psychological; Drug therapy; Practice Guideline.

\section{Introducción}

El uso de medicamentos es una estrategia frecuente para control de los problemas de conducta de las personas con DI. Su aplicación requiere que continuamente se valore su necesidad, eficacia y seguridad. Cuando se trata de problemas de conducta en personas con DI, si consideramos la carencia de investigación sobre el tema y que las actuales recomendaciones terapéuticas se basan tan solo en opiniones de expertos, los estudios observacionales cobran una especial importancia.

Las personas con DI son aquellas cuya función intelectual está por debajo de la población general, unido generalmente a un déficit en la conducta de adaptación al entorno. Se dice que existe un "déficit de adaptación" cuando los individuos no satisfacen la norma de independencia personal y responsabilidad social, esperada para su edad o grupo cultural (1). Actualmente el énfasis se pone en la interacción individuo-ambiente y no clasifican al sujeto en función del coeficiente intelectual (CI) sino atendiendo al apoyo que necesita la persona para desenvolverse en un entorno dado (2-3). La prevalencia promedio mundial de retaso mental estimada es de 10,37 casos por 1000 habitantes (4). En España se estima que la prevalencia de DI es de aproximadamente 500.000 personas (1\% de la población), con una tasa de crecimiento de 10.000 individuos/año, aproximadamente el $4 \%$ de los hogares del país están afectados (5). Se conocen más de 250 causas biológicas, pero en aproximadamente solo el $25 \%$ de las personas con DI se ha identificado una causa biomédica. En el 75\% restante se desconoce la causa, o se deben a factores psicosociales. La DI leve es generalmente idiopática o familiar, pero las formas graves y profundas son normalmente genéticas o relacionadas con una lesión cerebral. Tanto los factores hereditarios, como los mecanismos ambientales, incluyendo los socio-culturales, pueden contribuir a la transmisión familiar del DI leve a lo largo de generaciones (6-7).

Los estudios epidemiológicos han mostrado una tasa de morbilidad psiquiátrica (esquizofrenia, depresión, trastorno obsesivo-compulsivo...), en la población con DI, entre $8 \%$ y $15 \%$; y hasta el $50 \%$ cuando se incluye trastornos de conducta (8-10). Se llama trastorno de conducta a aquella conducta culturalmente anormal de tal intensidad, frecuencia o duración que puede poner en grave peligro la se- 
ORIGINALES Y REVISIONES

guridad física de la persona o de otros, o conducta que puede limitar el uso de los servicios habituales de la comunidad o hacer que le nieguen el acceso a los mismos a la persona (11).

Las deficiencias comunicativas y cognitivas presentes en muchos sujetos con discapacidad intelectual son de tal magnitud que dificultan enormemente el diagnóstico psiquiátrico, que ha de basarse únicamente en correlatos biológicos y conductuales. Existen desacuerdos sobre la relación entre psicopatología y discapacidad intelectual, y de la aplicabilidad de los actuales sistemas de clasificación y evaluación psiquiátrica a este grupo de población (12-13). El "Inventory for client and agency planning" (ICAP) recomendado por la American Association of Mental Retardation (AAMR) es un instrumento cuyo principal propósito es ayudar a la valoración y evaluación de servicios a las personas con DI. Sus características técnicas y facilidad de aplicación lo hacen útil tanto para pequeñas como para grandes poblaciones así como para todos los grupos de edad y variedad de habilidades. Existe una versión española estandarizada (14). Las personas con deficiencia mental constituyen una de las poblaciones más medicadas con psicofármacos, recibiendo neurolépticos entre $1 / 3$ y $3 / 4$ de los sujetos con este diagnóstico. Se argumenta como razón para ello la alta frecuencia y gravedad de los trastornos de conducta presentes en esta población (15-16). La elevada prescripción de psicofármacos en personas con DI es motivo de preocupación porque se carece de suficiente soporte empírico, están fuertemente influidas por factores no médicos, pueden originar graves problemas iatrogénicos. Ello en un contexto de creciente reivindicación de los derechos y necesidades de los discapacitados (17-18).

En la actualidad existe un consenso de expertos que pretende guiar la toma de decisiones farmacoterapéuticas en sujetos con DI y problemas conductuales (19). Es de suponer que el seguimiento de estas recomendaciones debería conducir a una mayor eficacia del tratamiento (20), que se manifestaría en una reducción de los problemas conductuales sin merma (o incluso mejoría) de las habilidades adaptativas. A pesar de ello se ha detectado disparidad de opinión al respecto entre psiquiatras prácticos y expertos en psicofarmacoterapia (21).

El objetivo del presente trabajo es: 1) describir la práctica real de la farmacoterapia de un grupo de sujetos con DI; 2) compararla con las recomendaciones de un grupo de expertos; y 3) tratar de determinar en que medida el alejamiento de estas recomendaciones se refleja en la intensidad de los problemas conductuales y las habilidades adaptativas. 
ORIGINALES Y REVISIONES

\section{Material y métodos}

Se efectuó un estudio observacional, transversal y cuantitativo, en un colectivo de sujetos diagnosticados de DI según los criterios CIE-10, asistidos en el Centro San Rafael en Alicante. La observación se realizó durante el verano del año 2005. Se registró el tratamiento farmacológico vigente de cada sujeto en el momento del estudio. Los medicamentos se han clasificado según el sistema "Anatómico Terapéutico Químico" (ATC) y las dosis recibidas se han cuantificado en "Dosis Diarias Definidas" (DDD) (22).

El tratamiento recibido por cada sujeto se comparó con las recomendaciones de los expertos (19) en lo referente a 1) indicación del fármaco,2) dosis empleadas; 3) duración del tratamiento y 4) polifarmacia; según los siguientes criterios operativos.

1) Indicación: Se considera que cumple el criterio de indicación de un fármaco cuando en la guía viene especificado como tratamiento de $1^{\circ}$ elección para el trastorno psiquiátrico o problema de conducta que padece el sujeto, o en caso de estar especificado como de $2^{\circ}$ elección, hay constancia en la historia clínica de resistencia o intolerancia al tratamiento de $1^{\circ}$ elección (19).

2) Dosis: Se considera cumplido el criterio de dosis si la que recibe el paciente está incluida dentro de los intervalos indicados para cada fármaco. También se considerará cumplido si siendo la dosis inferior al intervalo recomendado, el paciente ha sido adecuadamente controlado, o requiere un incremento más lento de la dosis para evitar intolerancia y así consta en la historia clínica. Si la dosis fuera superior al intervalo terapéutico, pero en la historia clínica hubiera constancia de un control insuficiente con dosis menores y una tolerancia adecuada entonces también se considerará cumplido el criterio. En los casos en que no se den las circunstancias descritas anteriormente, el criterio de dosis se considerará incumplido (19).

3) Duración de la pauta actual: Se considerará inadecuado cualquier cambio de tratamiento sin haber dejado transcurrir el tiempo mínimo de 3 semanas para los antipsicóticos, 1 semana para los estabilizadores del humor y 6 semanas para los antidepresivos ISRS; excepto durante la titulación de dosis al inicio del tratamiento, hasta alcanzar la dosis de mantenimiento, periodo durante el cual es adecuado hasta dos cambios en 1 semana. Si el paciente se encuentra estabilizado, se considerará que al menos debe recibir una revisión semestral actualizando el tratamiento completo (19).

4) Polifarmacia: El uso de dos fármacos de la misma clase terapéutica (dos antidepresivos o dos antipsicóticos) está raramente justificado; sin embargo el uso de dos o más fármacos de diferente clase terapéutica es apropiado en determinadas situaciones como la depresión psicótica o la depresión bipolar y en caso de respuesta parcial al tratamiento de elección. Se considera incumplido el criterio de 
ORIGINALES Y REVISIONES

polifarmacia cuando se utilizan simultáneamente dos psicofármacos fuera de las recomendaciones indicadas anteriormente (19).

Se ha tabulado el grado de DI y los diagnósticos psiquiátricos y problemas conductuales siguiendo la guía (19). Las discapacidades, distintas a la DI y/o enfermedad mental se han tabulado como motóricas, sensoriales o del habla. Todos los trastornos epilépticos se han clasificado conjuntamente en una sola categoría. Se ha tabulado la frecuencia de uso de cada grupo de psicofármacos, según el criterio que se indica a continuación: antipsicóticos, grupo N05A, excepto litio; antidepresivos, grupo N06A; anticonvulsivantes y estabilizadores del humor N03 y litio; ansiolíticos e hipnóticos grupos N05B y N05C (22). Las edades y el tiempo de institucionalización se han expresado en años y las dosis acumuladas de cada grupo terapéutico anterior se expresaron en DDD (22).

Los problemas de conducta se miden con el índice general normalizado de problemas de conducta, que puede oscilar desde +10 hasta -70 . Se consideran valores dentro de la normalidad a las puntuaciones iguales o superiores a -10 ; de -11 a -20 se considera que la conducta es levemente problemática; de -21 a -30 se considera moderadamente problemática; de -31 a -40 grave y las puntuaciones $<-40$ reflejan problemas de conducta muy graves o críticos. Las conductas adaptativas se expresan en la edad equivalente en meses (13).

Las variables de escala se describieron indicando media, desviación típica, mediana, cuartiles $1^{\circ}$ y $3^{\circ}$, mínimo y máximo. Las variables categóricas u ordinales se describieron como frecuencias absolutas y porcentajes.

Para cada recomendación de la guía se ha determinado la fracción de sujetos que la cumplía. Se han contrastado los valores de los problemas de conducta y las conductas adaptativas que se expresan en la edad equivalente en meses obtenidos con el ICAP en función del cumplimiento o no de cada una de las recomendaciones de tratamiento, para ello se ha empleado la prueba estadística U de Mann-Whitney (23). Se considera estadísticamente significativa p-bilateral $<0,05$.

\section{Resultados}

La población estudiada estuvo compuesta por 19 mujeres y 41 hombres, con edades comprendidas entre 20 y 89 años, con DI de moderado a profundo. La frecuencia de padecimiento adicional de problemas médicos o discapacidades físico-sensoriales y de epilepsia, y de diagnóstico psiquiátrico-conductual añadidos a la DI es alta. Casi la mitad presentaba problemas de conducta calificados de moderados a muy graves. Por termino medio los sujetos de la muestra presentaron habilidades adaptativas de magnitud similar a las que presenta un niño de 32 meses 
(tabla 1). La Tabla 2 informa sobre la frecuencia de diagnósticos psiquiátricos y los problemas de conducta que se encontraron en esta población.

El $90 \%$ de los sujetos tomaba algún tipo de medicamento en el momento de la observación, siendo los medicamentos para el Sistema Nervioso Central (SNC) los más utilizados, seguidos a distancia por los medicamentos para el aparato digestivo y metabolismo, para el aparato cardiovascular y para la sangre y órganos hematopoyéticos. Entre los medicamentos para el SNC, los antipsicóticos fueron

Tabla 1.

Características demográficas y de discapacidad de la población estudiada.

\begin{tabular}{|c|c|c|c|c|c|c|c|}
\hline & media & D.T. & MIN. & MAX. & Q1 & MED. & $\mathrm{Q} 3$ \\
\hline Edad (años) & 48,4 & 13,4 & 20,0 & 89,0 & 39,0 & 47,0 & 56,8 \\
\hline Puntuaciones ICAP & media & D.T. & MIN. & MAX. & Q1 & MED. & Q3 \\
\hline $\begin{array}{l}\text { Índice general de problemas } \\
\text { de conducta }\end{array}$ & $-20,3$ & 13,7 & -62 & 1 & $-29,5$ & -20 & -8 \\
\hline $\begin{array}{l}\text { Conductas adaptativas en } \\
\text { edad equivalente en meses }\end{array}$ & 32,2 & 22,0 & 2 & 103 & 15,3 & 23,50 & 47,3 \\
\hline Sexo & $\mathrm{N}^{\mathrm{o}}$ & $\%$ & & & & & \\
\hline Varones & 19 & 32 & & & & & \\
\hline Mujeres & 41 & 68 & & & & & \\
\hline Grado de deficiencia mental & $\mathrm{N}^{\mathrm{o}}$ & $\%$ & & & & & \\
\hline moderado & 6 & 10 & & & & & \\
\hline grave & 23 & 38 & & & & & \\
\hline profundo & 31 & 52 & & & & & \\
\hline Otras discapacidades asociadas & $\mathrm{N}^{\mathrm{o}}$ & $\%$ & & & & & \\
\hline Epilepsia & 16 & 27 & & & & & \\
\hline $\begin{array}{l}\text { Trastorno psiquiátrico o } \\
\text { problema de conducta }\end{array}$ & 50 & 83 & & & & & \\
\hline $\begin{array}{l}\text { Problemas médicos o } \\
\text { discapacidad física o } \\
\text { sensorial }\end{array}$ & 52 & 87 & & & & & \\
\hline $\begin{array}{l}\text { Gravedad de los problemas de } \\
\text { conducta (categorización del } \\
\text { índice general de problemas de } \\
\text { conducta) }\end{array}$ & $\mathrm{N}^{\mathrm{o}}$ & $\%$ & & & & & \\
\hline normal & 20 & 34 & & & & & \\
\hline leve & 12 & 20 & & & & & \\
\hline moderado & 14 & 23 & & & & & \\
\hline grave & 9 & 15 & & & & & \\
\hline muy grave & 5 & 8 & & & & & \\
\hline
\end{tabular}

D.T. desviación típica. MIN: mínimo. MAX: máximo. Q1: primer cuartil. MED: mediana. Q3: tercer cuartil. ICAP: Inventory for client and agency planning. 
Tabla 2.

Número de sujetos con diagnósticos psiquiátricos y problemas conductuales.

\begin{tabular}{|l|c|c|}
\hline Diagnósticos psiquiátricos (CIE-10) & $\mathrm{N}$ & $\%$ \\
\hline ninguno & 28 & 47 \\
\hline trastornos de movimientos estereotipados & 6 & 8 \\
\hline trastorno profundo del desarrollo (espectro autista) & 5 & 7 \\
\hline psicosis no especificada & 4 & 3 \\
\hline trastorno bipolar & 3 & 3 \\
\hline hiperactividad por déficit de atención & 2 & 3 \\
\hline esquizofrenia & 2 & 3 \\
\hline trastorno de control de impulso & 2 & 2 \\
\hline trastorno del sueño & 1 & 2 \\
\hline trastorno obsesivo-compulsivo & 1 & 8 \\
\hline trastorno de ansiedad & 1 & 20 \\
\hline varios de los anteriores & 5 & 8 \\
\hline Problemas conductuales & $\mathrm{N}$ & 3 \\
\hline ninguno & 12 & 3 \\
\hline conducta auto o heteroagresivas o destructivas & 5 & 2 \\
\hline impulsividad /hiperactividad & 2 & 64 \\
\hline retraimiento social & 2 & 2 \\
\hline conducta oposicional / desobediencia & 1 & \\
\hline varias de las anteriores & 38 & \\
\hline
\end{tabular}

los más empleados, mientras que ningún sujeto tomaba antidepresivos (tabla 3). Nueve sujetos toman anticonvulsivantes sin padecer epilepsia. Nueve sujetos toman fármacos para el SNC no clasificables dentro de las categorías mencionadas. La tabla 4 informa de la distribución de las dosis (expresadas como DDD/sujetodía) de los distintos subgrupos de medicamentos para el SNC. Puede observarse que las dosis medias de los tres grupos de psicofármacos indicados son próximas a la unidad, sin embargo la distribución de las dosis de antipsicóticos es extraordinariamente asimétrica, mostrando que la mayoría de los pacientes reciben dosis bajas o muy bajas, mientras que unos pocos reciben dosis notablemente altas. Las dosis de antiepilépticos, cuando se emplean para tratar la epilepsia son mayores que cuando se emplean para otros usos.

Los resultados de la evaluación del cumplimiento con las recomendaciones de los expertos en farmacoterapia, que se aplicaron solo a los 43 sujetos que recibían psicofármacos y/o antiepilépticos sin diagnóstico de epilepsia, se indican en la tabla 5. Los criterios de indicación y de duración fueron con diferencia los más satisfechos, $86 \%$ y 95\% respectivamente; el cumplimiento de los criterios de dosis y polifarmacia superó el 55\%. De los 19 sujetos que incumplen el criterio de dosis, 7 emplean dosis superiores al rango recomendado e inferior en 12. De los 19 sujetos que incumplen el criterio de polifarmacia, 10 reciben más de un fármaco del mismo grupo, mientras que 6 reciben varios fármacos de grupos distintos, y 3 reciben ambos tipos de politerapia. 
Tabla 3.

Número de sujetos usuarios de las distintas categorías de medicamentos.

\begin{tabular}{|c|c|c|}
\hline Grupos de medicamentos más utilizados $\left(1^{\circ}\right.$ nivel de la categoría ATC) & $\mathrm{N}^{\mathrm{o}}$ usuarios & $\mathrm{N}^{\mathrm{o}}$ usuarios \\
\hline A; aparato digestivo y metabolismo & 23 & 23 \\
\hline B; sangre y órganos hematopoyéticos & 8 & 8 \\
\hline C; aparato cardiovascular & 14 & 14 \\
\hline D; dermatológicos & 0 & 0 \\
\hline G; aparato genitourinario y hormonas sexuales & 1 & 1 \\
\hline $\mathrm{H}$; terapia hormonal, excluidas hormonas sexuales & 0 & 0 \\
\hline $\mathrm{J}$; terapia antiinfecciosa & 1 & 1 \\
\hline L; antineoplásicos; inmunomoduladores & 0 & 0 \\
\hline M; aparato locomotor & 2 & 2 \\
\hline N; Sistema Nervioso Central & 45 & 45 \\
\hline P; antiparasitarios, insecticidas, repelentes & 0 & 0 \\
\hline $\mathrm{R}$; aparato respiratorio & 2 & 2 \\
\hline $\mathrm{S}$; órganos de los sentidos & 0 & 0 \\
\hline V; otros medicamentos & 0 & 0 \\
\hline \multicolumn{3}{|l|}{ Subgrupos de medicamentos para el SNC (N) } \\
\hline antiepilépticos, N03 & 24 & 24 \\
\hline antipsicóticos (N05A, excepto litio) & 34 & 34 \\
\hline litio & 1 & 1 \\
\hline ansiolíticos y/o hipnóticos (N05B y/o N05C) & 9 & 9 \\
\hline Antidepresivos (N06A) & 0 & 0 \\
\hline algún psicofármaco (N05 y/o N06A) & 38 & 38 \\
\hline litio y/o antiepilépticos & 25 & 25 \\
\hline antiepilépticos (sin epilepsia) y/o psicofármacos & 43 & 43 \\
\hline Otros fármacos para el SNC & 9 & 9 \\
\hline
\end{tabular}

Tabla 4.

Distribución de dosis (DDD/sujeto x día) de cada subgrupo de fármacos para el SNC, empleadas en los sujetos que los utilizan.

\begin{tabular}{|l|c|c|c|c|c|c|c|c|}
\hline Categoría de fármacos & $\mathrm{N}^{\mathrm{o}}$ & media & D.T. & MIN. & MAX. & Q1 & MED & Q3 \\
\hline antipsicóticos (N05A, excepto litio) & 34 & 0,94 & 0,94 & 0,04 & 3,75 & 0,16 & 0,69 & 1,33 \\
\hline $\begin{array}{l}\text { ansiolítico-hipnóticos (N05B y/o } \\
\text { N05C) }\end{array}$ & 9 & 1,17 & 1,03 & 0,13 & 3,00 & 0,21 & 1,20 & 2,00 \\
\hline litio y/o antiepilépticos sin epilepsia & 8 & 0,89 & 0,85 & 0,13 & 2,85 & 0,30 & 0,75 & 0,95 \\
\hline antiepilépticos (solo epilépticos) & 16 & 1,87 & 0,92 & 0,33 & 3,60 & 1,32 & 1,66 & 2,56 \\
\hline
\end{tabular}

$N^{o}$ : Número de Usuarios. D.T: Desviación típica. MIN: Mínimo. MAX: Máximo. Q1: primer cuartil. MED: mediana. Q3: tercer cuartil.

Tabla 5.

Cumplimiento del criterio entre los 43 sujetos usuarios de anticonvulsivantes ( in ser epilépticos) o antipsicóticos.

\begin{tabular}{|l|c|c|}
\hline \multirow{2}{*}{} & \multicolumn{2}{|c|}{ Frecuencia de conformidad con los criterios } \\
\cline { 2 - 3 } & $\mathrm{N}^{\circ}$ de sujetos & $\%$ \\
\hline Criterio de indicación & 37 & 86,0 \\
\hline Criterio de dosis & 24 & 55,8 \\
\hline Criterio de duración & 41 & 95,3 \\
\hline Criterio de polifarmacia & 24 & 55,8 \\
\hline
\end{tabular}


Tabla 6.

Significación de las diferencias de las puntuaciones del índice general de problemas de conducta y de las edades en meses equivalentes a las conductas adaptativas de los sujetos, en función de que el tratamiento farmacológico haya seguido o no, las recomendaciones indicadas en la guía de expertos para los 4 criterios seleccionados.

\begin{tabular}{|c|c|c|c|c|c|c|c|c|}
\hline & \multicolumn{2}{|c|}{$\begin{array}{l}\text { Criterio de } \\
\text { indicación }\end{array}$} & \multicolumn{2}{|c|}{$\begin{array}{c}\text { Criterio de } \\
\text { dosis }\end{array}$} & \multicolumn{2}{|c|}{$\begin{array}{c}\text { Criterio de } \\
\text { duración }\end{array}$} & \multicolumn{2}{|c|}{$\begin{array}{c}\text { Criterio de } \\
\text { polifarmacia }\end{array}$} \\
\hline & 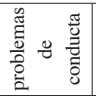 & 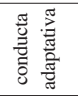 & 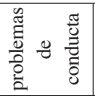 & 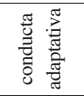 & 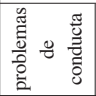 & 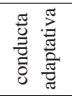 & 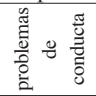 & 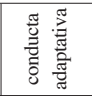 \\
\hline U de Mann-Whitney & 76,00 & 97,00 & 218,00 & 141,00 & 6,00 & 38,50 & 112,00 & 227,50 \\
\hline Z & $-1,228$ &,- 491 &,- 245 & $-2,129$ & $-2,020$ &,- 144 & $-2,839$ &,- 012 \\
\hline Sig. asintót. (bilateral) (p) & ,220 & 623 & ,807 & ,033(*) &, $043(*)$ & 885 &, $005(*)$ & 990 \\
\hline
\end{tabular}

(*) $p<0,05$, diferencias estadísticamente significativas.

Al contrastar los índices de problemas de conducta y la edad equivalente en meses a la conducta adaptativa en función del cumplimiento o no de cada uno de los 4 criterios, indicación, dosis, duración y polifarmacia, se encontró que el cumplimiento del criterio de dosis se asoció significativamente con mejor conducta adaptativa, mientras que el cumplimiento de los criterios de duración y polifarmacia se asociaron con menores problemas de conducta. No hubo asociación entre el cumplimiento de criterio de indicación con la intensidad de los problemas de conducta, ni con las habilidades adaptativas. La figura 1 muestra los diagramas de cajas correspondientes, y la tabla 6 los resultados de la prueba U de Mann-Whitney para la significación estadística.

\section{Discusión}

Los resultados obtenidos pueden considerarse como una validación de las recomendaciones por consenso de expertos para el tratamiento de los problemas de conducta en personas con DI (19). La metodología empleada ya había sido utilizada por nosotros al evaluar la relación entre estado mental de un grupo de sujetos con esquizofrenia y el apego a un conjunto de criterios similares de calidad tratamiento farmacológico basado en datos (24).

El colectivo estudiado constituye un grupo particular de personas con DI, institucionalizados en un determinado centro. Es muy probable que los problemas conductuales y los efectos de los fármacos empleados para tratarlos, así como los recursos terapéuticos alternativos a estos de que disponen sean muy diferentes de los que encontraríamos en otras subpoblaciones de sujetos con DI, por ejemplo niños que conviven con sus padres. Por tanto nuestros resultados solo son aplicables y comparables con los de colectivos similares a los que hemos estudiado. 
Figura 1.

Diagramas de caja para la distribución de las puntuaciones de índice general de problemas de conducta y de las edades en meses equivalentes a las habilidades adaptativas de los sujetos, en función de que el tratamiento farmacológico haya seguido las recomendaciones indicadas en la guía de expertos para los 4 criterios seleccionados.

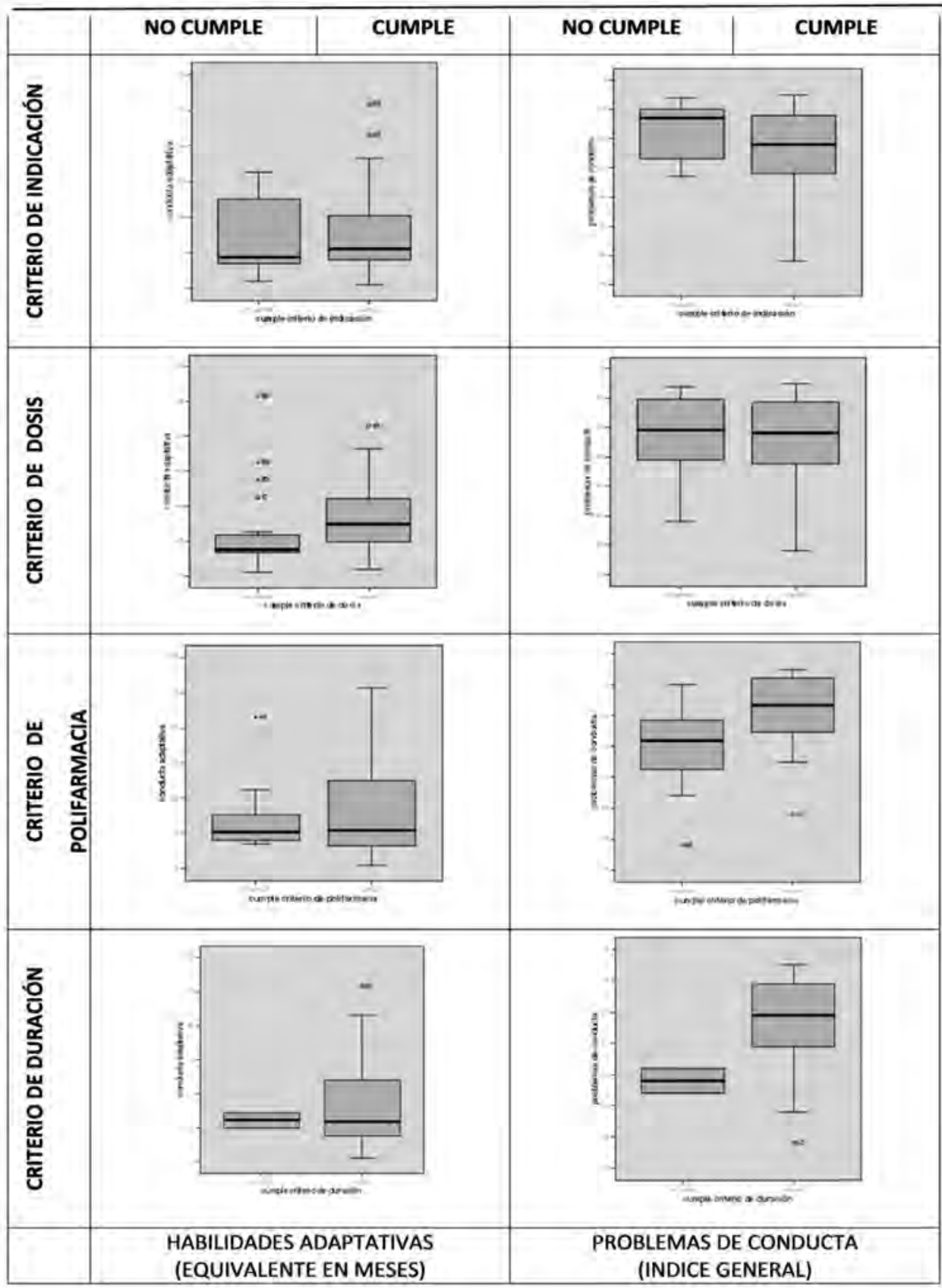


ORIGINALES Y REVISIONES

Los datos se recogieron en junio del 2005, dentro de un programa de mejora continua de la calidad, como un nuevo método de evaluación asistencial. A la dirección del centro se entregó un informe detallado de los resultados del estudio, cuyo resumen acabamos de exponer. Consideramos que los resultados son de interés, ya que pueden servir como datos basales para futuros estudios de calidad de prescripción en este mismo centro, y como referencia para comparación en caso de estudios similares en otros centros.

El colectivo estudiado es un grupo de sujetos con DI que requiere institucionalización, generalmente por razones de índole social, por la carencia de habilidades adaptativas y la presencia de problemas conductuales. Muchos de estos sujetos presentan discapacidades física o sensorial. Los trastornos convulsivos constituyen también una comorbilidad frecuente. Esta descripción es similar a la encontrada por otros cuando se revisa las necesidades médicas de personas con DI que recibe cuidados en instituciones $(8,25-28)$.

$\mathrm{Al}$ igual que en nuestros resultados, en otros estudios también se ha descrito una elevada frecuencia de uso de medicamentos para el SNC, medicamentos para el aparato digestivo (laxantes y de medicamentos para el reflujo gastroesofágico) (29-30) y de medicamentos para el sistema cardiovascular (31). Ello es coherente con los problemas de salud prevalentes en este colectivo (32-33).

Entre nuestros resultados aparece un elevado empleo de psicofármacos, más del $72 \%$ de los sujetos reciben fármacos para problemas psiquiátrico-conductuales (psicofármacos y/o anticonvulsivantes sin padecer epilepsia), siendo los antipsicóticos los psicofármacos más frecuentemente utilizados, 57\% de los sujetos los reciben. Estas cifras, si bien están dentro de las frecuencias más altas observadas en otros centros para este tipo de población (34-35) y son bastante superiores a las encontradas previamente en este mismo centro (27-28). Otros autores han descrito incrementos con el tiempo en la frecuencia de uso de psicofármacos en colectivos de personas con DI (34-36). Vivir en un entorno residencial y la edad eran factores asociados de forma significativa a una mayor probabilidad de que un sujeto con DI fuera tratado con antipsicóticos (15).

Se carece de pruebas científicas acerca del beneficio o perjuicio de la administración de antipsicóticos en las personas con DI para la corrección o mejoría de los problemas de conducta (37). Así mismo tampoco existen estudios de que los pacientes esquizofrénicos con DI se beneficien de los efectos de los antipsicóticos (38) a pesar de ser una de las poblaciones más medicadas con ellos. Otros estudios (39) en los que se compara risperidona y placebo para el tratamiento de conductas agresivas en individuos con DI, muestran beneficios con el fármaco activo, pero a costa de efectos adversos importantes. Cuando se ha examinado en un ensayo doble ciego controlado, la efectividad de los antipsicóticos: haloperidol, risperidona y ambos asociados frente a placebo, para el control de conductas agresivas en sujetos 
con DI, los resultados favorecieron al grupo placebo (40). Por tanto la elevada frecuencia del uso de antipsicóticos en los individuos con DI es una cuestión de creencias más que de evidencias.

El uso de anticonvulsivantes es después de los antipsicóticos, la categoría de medicamentos más empleada en este colectivo. La frecuencia de su empleo sobrepasa a la frecuencia de sujetos con diagnóstico de epilepsia, ya que algunos de los fármacos de esta categoría se emplean en el tratamiento y prevención de episodios afectivos, en el dolor neuropático y otras indicaciones como problemas de conducta relacionados con dificultades en el control de impulsos (41-42). Aunque su efectividad en este ultimo uso no está claramente establecida (43), si se ha observado que el uso de anticonvulsivantes ha llevado aparejado un descenso en el uso de psicofármacos (42).

Ninguno de los pacientes de la población estudiada recibe antidepresivos. Otros estudios también han encontrado un uso infrecuente de antidepresivos (15), se ha observado que los antidepresivos pueden deteriorar el estado de este tipo de pacientes (44-45). Los médicos prácticos suelen valorar la utilidad de los antidepresivos más que los expertos en psicofarmacología (46), por tanto este hallazgo es coherente con el apego a las recomendaciones de estos últimos (19). En el grupo y en el momento estudiado sólo había un sujeto que recibía litio, probablemente por dificultades en controlar y mantener niveles plasmáticos adecuados. También las benzodiazepinas se han relacionado con un empeoramiento de los problemas conductuales hasta en un 10-25\% de las personas con DI que las reciben, además este empeoramiento es fácilmente confundido con otros problemas de conducta o patologías psiquiátricas (46).

En el grupo de sujetos estudiados, las dosis medianas de las categorías de medicamentos de interés, se encuentran en torno a una DDD, siendo algo menores para los antipsicóticos y para los antiepilépticos cuando se emplean en sujetos sin diagnóstico de epilepsia. Las dosis medianas son algo mayores para ansiolítico - hipnóticos y para antiepilépticos empleados en sujetos con diagnóstico de epilepsia, probablemente reflejando el uso simultáneo en un sujeto de un ansiolítico y un hipnótico y el uso de polifarmacia anticonvulsivante en sujetos con crisis de difícil control. No obstante también puede observarse que algunos pocos sujetos llegan a tomar dosis muy altas (como muestran las dosis máximas empleadas). Un estudio (47) mostró que la respuesta más probable a la aparición, en sujetos con DI, de síntomas médicos y conductuales que pueden ser potenciales efectos adversos a alguno de los psicofármacos que reciben y que están descritos como tales en las fichas técnicas de los medicamentos, es el incremento de las dosis del mismo o la adición de un nuevo fármaco, en lugar de la reducción de dosis o la retirada del mismo, la adición de un nuevo fármaco. Por otra parte se ha asociado el uso de dosis altas a la práctica de la polifarmacia (48). 
ORIGINALES Y REVISIONES

Los tratamientos psicofarmacológicos empleados en la muestra estudiada siguen las recomendaciones del panel de expertos (19) en la mayoría de los casos, al menos en cuanto a las características examinadas (indicación, dosis, duración y polifarmacia). Además los tratamiento que eran conforme a dos (duración y polifarmacia) de los cuatro criterios se asociaron estadísticamente con menores índices generales de problemas de conducta; y los conformes a un tercer criterio (dosis) se asociaron a mayor edad en meses equivalentes a las conductas adaptativas de los sujetos. Estos resultados apoyan la hipótesis planteada: El seguimiento de las recomendaciones del grupo de expertos mejora el resultado terapéutico (reduciría las conductas problemáticas, a la vez que las conductas adaptativas no empeoran). Debemos ser conscientes que el diseño de nuestra observación, un estudio transversal, presenta las limitaciones inherentes al mismo. Efectúa un "retrato instantáneo", pero no puede captar el sentido dinámico de las consecuencias de la intervención, para lo que sería necesario la realización de estudios longitudinales. Aunque no hemos encontrado diferencias en algunas de las comparaciones efectuadas, esto puede deberse a falta de potencia estadística (capacidad para confirmar que dos tratamientos aparentemente iguales en realidad lo son), para lo que serían necesario aumentos del tamaño de la muestra, lo que está fuera del alcance del presente estudio, que se ha limitado a un escrutinio en un centro concreto. Por ejemplo un criterio elemental para valorar la idoneidad de uso de un medicamento es su indicación. En nuestro estudio el criterio de indicación no mostró diferencias en el resultado entre los que lo cumplían y no, esto puede deberse a que la mayoría de los sujetos lo cumplían y también a que los sujetos con respuestas inadecuadas reciben tratamientos alternativos, algunos de los cuales responden a las mismas, reduciendo la posibilidad de observar diferencias entre grupos. Dentro del grupo estudiado encontramos sujetos con problemas de conducta y/o diagnóstico psiquiátrico a los cuáles se les administra psicofármacos y otros en las mismas condiciones a los cuáles no se les administra psicofármacos. Es posible que los pacientes que recibían psicofármacos fueran aquellos con problemas de conducta más graves o resistentes a las medidas no farmacológicas (50).

Existen pocos estudios sobre problemas de conducta y diagnósticos psiquiátricos y los que hay presentan sesgos metodológicos importantes y en muchas ocasiones los únicos datos de los que se disponen proceden de estudios hechos en personas que no padecen DI, y esta extrapolación no está exenta de riesgos (50). El uso de medicamentos psicotrópicos en personas con DI es un problema controvertido que ha recibido mucha atención. Hay preocupación en relación con la falta de selectividad en su eficacia y con los efectos secundarios que pueden producir. La evidencia sugiere que muchos profesionales de la salud que se han especializado en cuidados del paciente con DI, necesitan mantenerse actualizados con relación a los problemas que subyacen al uso de tales medicamentos: eficacia, efectos se- 
cundarios e interacciones. Todos los miembros del equipo multidisciplinar deben y necesitan comprender claramente las razones por las que se prescriben estos medicamentos, especialmente cuando se usan de una forma sintomática en el tratamiento de los problemas conductuales, y formar parte activa de los procesos de revisión de uso de medicamentos (51).

\section{Conclusiones}

1. Casi todos los sujetos de este estudio (90\%) reciben algún tipo de fármaco, los más utilizados son medicamentos para el SNC y dentro de este grupo los antipsicóticos.

2. En general, en el centro estudiado, la prescripción de psicofármacos se ajusta a las recomendaciones de los expertos.

3. Las situaciones de incumplimiento más frecuentes consisten en el uso de varios medicamentos del mismo grupo (polifarmacia) y /o en el uso de dosis fuera del rango recomendado.

4. El seguimiento de las recomendaciones de expertos parece reflejarse en menores índices de problemas conductuales y mejores habilidades adaptativas.

5. Una actitud activa del equipo multidisciplinar que asiste al paciente con DI y evalúa el uso de fármacos (idoneidad, eficacia y seguridad) es fundamental.

BIBLIOGRAFÍA:

(1) CIE-10. Trastornos mentales y del comportamiento, descripciones clínicas y pautas para el diagnóstico. Organización Mundial de la Salud, Meditor, Madrid. 1992

(2) American Association on Mental Retardation.'Mental Retardation: definition, classification, and systems of supports. 9a ed, American Association on Mental Retardation, Washington DC. 1992.

(3) Salvador-Carulla LS, Reed GM, Vaez-Azizi LM, Cooper SA, Martínez-Leal R, Bertellli M, Adnams C, Cooray S, Deb S, Dirani LA, Girimaji SC, Katz G, Kwok H, Luckasson R, Simeosson R, Walsh C, Munir K, Saxena S. Intellectual developmental disorders: towards a new name, definition and framework for "mental retardation/intellectual disability" in ICD-11. World Psychiatry. 2011; 10: $175-180$.

(4) Maulik PK, Mascarenhas MN, Mathers CD, Dua T, Saxena S. Prevalence of intellectual disability: a meta-analysis of population-based studies. Res Dev Disabil. 2011; 32:419-36.

(5) Bueno M. Mental deficiency, concept, epidemiology and preventive measures", en Seva A. "European Handbook of Psychiatry and Mental Health".Antropos editorial, Zaragoza, 1991.

(6) Katz G, Lazcano-Ponce E. Discapacidad intelectual: definición, factores etiológicos, clasificación, diagnóstico, tratamiento y prognosis. Salud Publica Mex 2008;50 supl 2:S132-S141. 
(7) Popper ChW, Steingard RJ "Trastornos de inicio en la infancia, la niñez o la adolescencia", en Hales RE, Yudofsky SC y Talbott JA "Tratado de Psiquiatría”, $2^{\circ}$ ed, The American Psychiatric PressAncora SA, Barcelona. 1996.

(8) Almenara J, García R, Novalbos JP, Merello B, Abellan MJ, García C. Evaluación médica y psicosocial de una población adulta con discapacidad intelectual. Rev Esp Salud Públic. 1999; 73:383-392.

(9) Morgan VA, Leonard H, Bourke J, Jablensky A. Intellectual disability co-occurring with schizophrenia and other psychiatric illness: population-based study. Br J Psychiatry.2008 ;193:364-72.

(10) Campbell M, Malone RP. Mental retardation and psychiatric disorders. Hosp. Community Psychiatry, 1991; 42: 374-9.

(11) Emerson C. Challenging behaviour: Analysis and intervention in people with learning difficulties. Cambridge: Cambridge University Press, 1995.

(12) Salvador L. Discapacidad intelectual y enfermedad psiquiátrica: evaluación, tratamiento y servicios. Monografías en Neurociencias nº 4, Ed Neurociencias, Barcelona, 1995.

(13) García J, Feliu T, Usón M, Ródenas A, Aguilera F, Ramo R. Trastornos invisibles. las personas con discapacidad intelectual y trastornos mentales o de conducta. Siglo Cero, Revista Española sobre Discapacidad Intelectual 2009; 40, 230: 38-60.

(14) Montero D. Evaluación de la conducta adaptativa en personas con discapacidad, adaptación y validación del ICAP. Universidad de Deusto, Bilbao, 1993.

(15) Pol E, Ivorra J, Horga JF Aznar MT. Uso de fármacos por sujetos con retraso mental en Alicante (España). Farm Hosp 2001; 25:104-16.

(16) Stolker JJ, Heerdink ER, Leufkens HG, Clerkx MG, Nolen WA. Determinants of multiple psychotropic drug use in patients with mild intellectual disabilities or borderline intellectual functioning and psychiatric or behavioral disorders. Gen Hosp Psychiatry. 2001;23:345-9.

(17) Sachdev PS. Psychoactive drug use in an institution for intellectually handicapped persons. Med J Aust, 1991,155: 75-79.

(18) Holden B, Gitlesen JP.Psychotropic medication in adults with mental retardation: prevalence, and prescription practices. Res Dev Disabil. 2004; 25:509-21.

(19) Rush AJ, Frances A, eds. Expert Consensus Guideline Series: treatment of psychiatric and behavioral problems in mental retardation. Am J Ment Retard.2000, 105:159-226.

(20) Kissling W, Seemann V, Piwernetz K. "Quality management in psychiatry". Int Clin Psychopharmacol; 2001; 16(S3):S15-S24.

(21) Patel NC, Crismon ML, Rush AJ, Frances A. Practitioner versus Medication-Expert Opinión on Psychiatric Pharmacotherapy of Mentally Retarded Patients with Mental Disorders. Am J Health-Syst Pharm 2001; 58:1824-1829, 2001.

(22) "Anatomical Therapeutic Chemical(ATC) clasification index, including Defined Daily Doses(DDDs) for plain substances", Junary 2004; WHO Collaborative Center for Drug Stratistics Methodology,Oslo,2004.

(23) Norman GR, Streiner DL. Bioestadística. Mosby/Doyma Libros,Madrid 1996.

(24) Martinez-Granados F, Moñino-Martínez C, Pol-Yanguas E, Ivorra-Cano E, Villar-Malpica JL. Análisis de correlación entre la calidad de la prescripción de antipsicóticos y estado clínico en pacientes con esquizofrenia. Farm Hosp 2005; 29:95-103.

(25) Merrick J, Kandel I, Raskas M, Caplan M, Morad M. National survey 2004 on medical services for persons with intelectual disability in residential care in Israel. Int J Adolesc Med Healt 2010; 22:331-8 
(26) Merrick J, Kandel I, Lotan M, Aspler S, Fuchs BS, Morad M. National survey 2007 on medical services for persons with intelectual disability in residential care in Israel. Int J Adolesc Med Healt 2010; $22: 575-28$

(27) De-anta A, Martí B, Mata G, Pol E, Quesada JA, van-der-Hofstadt MD, Vegara ML. Uso de psicofármacos en sujetos adultos con retraso mental ingresados en dos centros distintos. Farm Hosp 1998; 22:23-24

(28) Calzado C, Carratalá A, Guarinos N, Moscardó C, Pol E, Quesada JA, Tarazona MV, Torras I, Torras I. Uso de psicofármacos en sujetos con retraso mental: factores asociados. Atención farmacéutica, 1999; 1:449-68.

(29) Van der Heide, van der Puten AA, van der Berg PB, Taxis K, VlaskampC. The documentation of health problems in relation to prescribed medication in people with profound intellectual and multiple disabilities. J Intellect Disabil Res 2009; 53:161-8

(30) Charlot L,Abend S, Ravin P, Mastis K, Hunt A, Deutsch C. Non-psychiatric health problems among psychiatric inpatients with intellectual disabilities. J. Intellect Disabil Res. 2011; 55:199-209.

(31) Walters RM. Prescribing requirements of elderly mentally handicapped: future demands on primary health care teams. J R Coll Gen Pract. 1988; 38:317-9

(32) Merrick J, Kandel I, Morad M. Health needs of adults with intelectual disability relevant for family physician. TheScientificWorldJournal 2003; 3:937-45.

(33) Sullivan WF, Berg JM, Bradley E, Cheetham T, Denton R, Heng J, Hennen B, Joyce D, Kelly M, Korrosy M, Lunsky Y, McMillan S. Primare care of adults with developmental disabilities. Canadian consensus guideline. Can Fam Physician 2011; 57:541-53)

(34) Singh NN, Ellis CR, Weschsler H. Psychopharmacoepidemiology of mental retardation: 1966 to 1955. J Child Adolesc Psychopharmacol. 1997; 7:255-66

(35) Merrick J, Kandel I, Stawki M. Trends in mental health services for people with intellectual disability in residential care in Israel 1998-2004. Isr J Psychiatry Relat Sci, 2006; 43:281-4

(36) Esbensen AJ, Greemberg JS, Seltzer MM, Aman MG. A Longitudinal Investigation of Psychotropic and Non-Psychotropic Medication Use Among Adolescents and Adults with Autism Spectrum Disorders. J Autism Dev Disord. 2009; 39: 1339-49.

(37) Brylewski J, Duggan L. Fármacos antipsicóticos para la conducta desafiante en personas con trastornos del aprendizaje. Revisión Cochrane traducida. En: La Biblioteca Cochrane Plus, 2005 Número 1. Oxford: Update Software Ltd. Disponible en: http://www.update-software.com. a fecha 20-04-05.

(38) Duggan L, Brylewski J. Antipsicóticos versus placebo para personas con esquizofrenia y dificultad del aprendizaje. Revisión Cochrane traducida. En: La Biblioteca Cochrane Plus, 2005 Número 1. Oxford: Update Software Ltd. Disponible en: http://www.update-software.com. a fecha 20-04-05.

(39) Young JG. Risperidone was effective for aggression in adolescents with disruptive behaviour disorders and below average intelligence. EBMH, 2002; 5:11.

(40) Tyrer P, Olivero-Africano P, Romeo P, Kanapp M, Dickens S, Bouras y otros. Neuroleptics in the treatment of aggressive challenging behaviour for people with intellectual disabilities: a randomized controlled trial (NACHBID). Health Technology Assessment 2009; 13 (21); accesible en www.hta.ac.uk, acceso a 15 del 7 del 2012.

(41) Janicak PG, Davis JM, Preskorn SH, Ayd FJ. Principles and Practice of Psychopharmacotherapy. Williams \& Wilkins; Baltimore, 1993.

(42) Leunissen CL, de la Parra NM, Tan IY, Rentmeester TW, Vader CI, Vendrik-Meekes MJ, ALdenkamp AP. Antiepileptic drugs with mood stabilizing properties and their relation with 
psychotropic drug use in institutionalized epilepsy patients with intellectual disability. Res Dev Disabil, $2011 ; 32: 2660-8$.

(43) Deb S, Chaplin R, Sohanpal SK, Unwing G, Soni R, Letrône L. The effectiveness of mood stabilizer and antiepileptic medication for the management of behaviour problems in adults with intellectual disability: a systematic review. J Intellect Disabil Res, 2008; 52: 107-13.

(44) Girimaji SC. Comorbidity of mental retardation and affective disorders. J Indian Med Assoc. 2000; 98:245,248-9

(45) Sohanpal SK, Deb S, Thomas C, Soni R, Lenôtre L, Unwing G. The effectiveness of antidepressant medication in the management of behaviour problems in adults with intelectual disabilities: a systematic review. J Intellect Disabil Res, 2007; 51: 750-65.

(46) Kalachnik JE, Hanzel TE, Sevenich R, Harder SR. Benzodiazepine behavioral side effects: review and implications for individuals with mental retardation. Am J Ment Retard. 2002;107:376-410.

(47) Valdovinos MG, Caruso M, Roberts C, Kim G, Kennedy CH. Medical and behavioral symptoms as potential medication side effects in adults with developmental disabilities. American Journal on Mental Retardation 2005; 110:164-70.

(48) Procyshyn RM, Honer WG, Wu TK, Ko RW, McIsaac SA, Young AH, Johnson JL, Barr AM. Persistent antipsychotic polypharmacy and excessive dosing in the community psychiatric treatment setting: a review of medication profiles in 435 Canadian outpatients. J Clin Psychiatry. 2010;71:566-73.

(49) Hassiotis A, Hall I. Intervenciones conductuales y cognitivo-conductuales para el comportamiento agresivo hacia el entorno en personas con dificultades de aprendizaje. Revisión Cochrane traducida. En: La Biblioteca Cochrane Plus, 2005 Número 1. Oxford: Update Software Ltd. Disponible en: http://www.update-software.com. a fecha 20-04-05.

(50) Matson JL, Bamburg JW, Mayville EA, Pinkston J, Bielecki J, Kuhn D, Smalls Y, Logan JR. Psychopharmacology and mental retardation: a 10 year review (1990-1999). Res Dev Disabil.2000; 21:263-96.

(51) Jenkins R. Use of psychotropic medication in people with a learming disability. $\mathrm{Br} \mathrm{J}$ Nurs.2000; 9:844-50.

(52) Novell R, Rueda P, Salvador L, Forgas E. Salud Mental y alteraciones de la conducta en las personas con discapacidad intelectual. Guía práctica para técnicos y cuidadores. Colección FEAPS. Madrid. 2003. 
